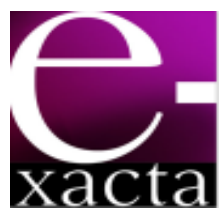

ISSN: 1984-3151

\title{
HieRARQUIA DA REDE URBANA DO VALE do MUCURI UTILIZANDO TÉCNICAS DE ANÁLISE MULTIVARIADA
}

\author{
URBAN NETWORK HIERARCHY OF MUCURI VALLEY USING \\ MULTIVARIATE ANALYSIS TECHNIQUES
}

\author{
1 Mestre em Geografia -Tratamento da Informação Espacial - \\ PUC Minas. Professor da rede municipal de ensino de \\ Betim-MG. flavioapl@yahoo.com.br. \\ 2 Doutor em Ciências em Informática. PUC Rio, RJ, 1988 \\ Professor do Programa de Pós-Graduação em Geografia- \\ Tratamento da Informação Espacial - PUC Minas. Belo \\ Horizonte, MG. lbarroso@pucminas.br. \\ 3 Pós doutorado em Geografia. Universidade Mc Gill, \\ Montreal, Canadá. 2007. Professor do Programa de Pós- \\ Graduação em Geografia-Tratamento da Informação \\ Espacial - PUC Minas. Belo Horizonte, MG. \\ dinizalexandre@terra.com.br.
}

Flávio Apolinário'; Leônidas Conceição Barroso²; Alexandre Magno Alves Diniz ${ }^{3}$

Recebido em: 30/08/2012 - Aprovado em: 10/11/2012 - Disponibilizado em: 30/11/2012

RESUMO: Neste artigo estuda-se a rede de cidades que compõem a Mesorregião do Vale do Mucuri, elaborando uma análise de como ela se encontra organizada e propondo, ainda, uma hierarquização de suas cidades por meio de sua tipologia, funcionalidade e complementaridade. Com métodos de análise espacial e uso de técnicas de estatística multivariada e de geoprocessamento, busca-se fazer um levantamento da oferta de serviços e da mobilidade demográfica nessas cidades, a fim de identificar níveis hierárquicos, analisados à luz das teorias geográficas.

PALAVRAS-CHAVE: Rede de cidade. Hierarquia Urbana. Geoprocessamento. Estatística Multivariada.

ABSTRACT: This paper aims to study the urban network of the Mucuri Valley Region. It is pointed out how the network is organized and proposed a ranking of their cities through their typology, functionality and complementarity. Spatial analysis methods and the use of multivariate statistical techniques and GIS allow a survey of the supply of services and population mobility in these cities in order to identify hierarchical levels, analyzed in the light of geographical theories.

KEYWORDS: Network of cities. Urban Hierarchy. GIS. Multivariate Statistics.

\section{INTRODUÇÃO}

Neste trabalho faz-se uma análise de como se encontra estruturada a rede de cidades da mesorregião do Vale do Mucuri, no Estado de Minas Gerais, e propõe-se uma hierarquização das cidades,por meio do estudo e interpretação dos dados, referentes à oferta de serviços e infraestrutura urbana, coletados para cada um dos vinte e três municípios, que compõem a referida mesorregião (Mapa1).

O estudo das redes urbanas (aí incluídas as noções de hierarquia, tipologia, posição geográfica e relacionamento das cidades) torna-se cada vez mais necessário nos dias de hoje, sobretudo para conhecimento regional, nacional e mundial, e para qualquer tipo de ação que se queira desenvolver sobe as cidades e as regiões. (AMORIM FILHO, 1990, p. 26). 


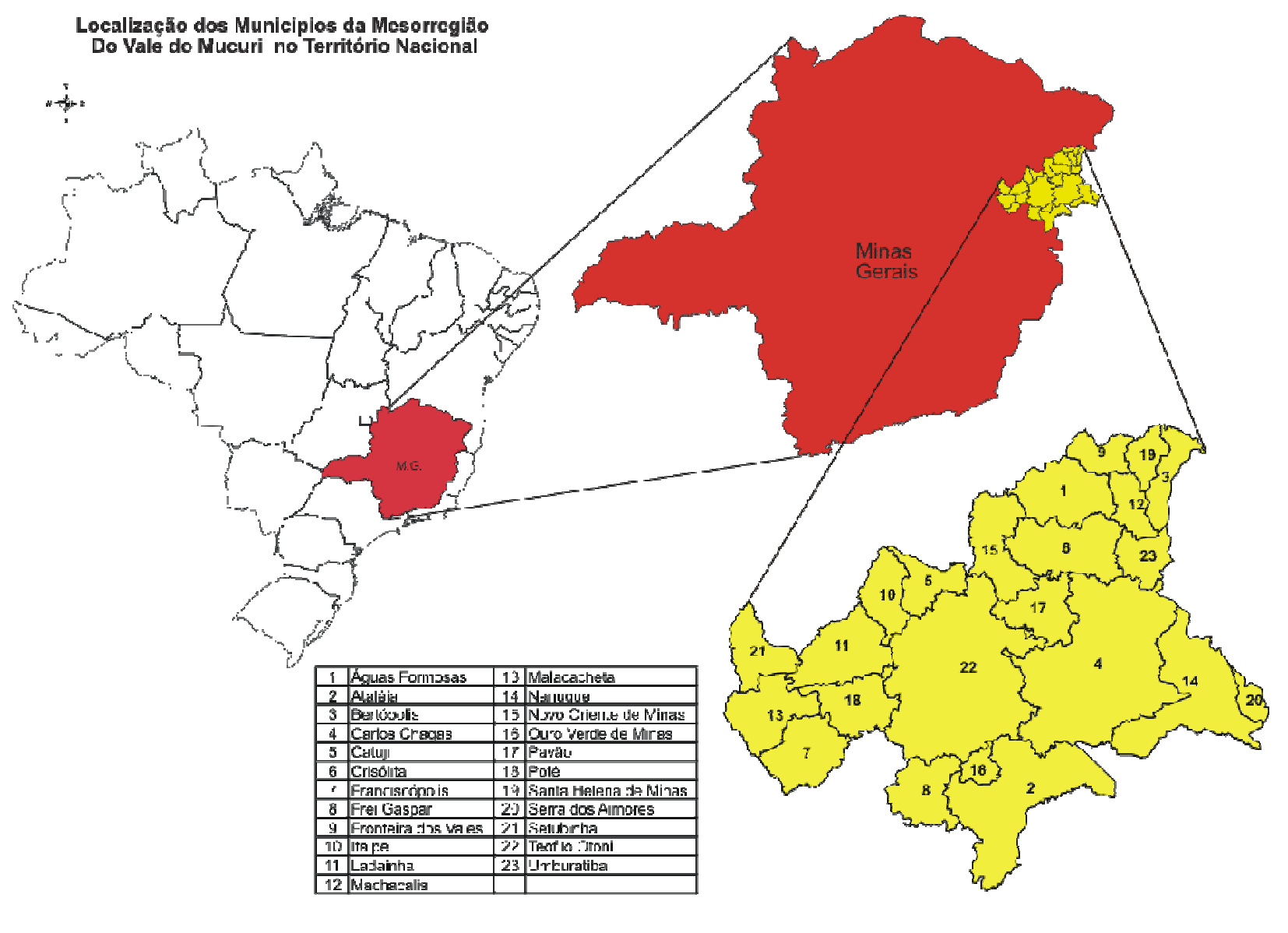

Organização: Flávio Apolinário, 2010
Lab. Estudos Urbanos e Regionais - PPGTIE - PUC MINAS Fonte:Geominas

Mapa 1: Localização dos municípios que compõem a Mesorregião do Vale do Mucuri.

Fonte: APOLINÁRIO, 2010.

Para investigar a organização da rede urbana da mesorregião do Vale do Mucuri foram utilizadas técnicas de estatística multivariada que auxiliaram na geração de um índice que mensurasse o grau de acessibilidade aos serviços urbanos, oferecidos por cada cidade da região estudada. A este índice chamou-se de I.A.U. (Índice de Acessibilidade Urbana).

\section{MÉTOdOS e TÉCNICAS}

\subsection{Coleta De dados}

Para determinar a hierarquia das cidades foi necessária a elaboração de um banco de dados dos municípios estudados, contendo informações sócioeconômicas e demográficas, bem como a existência ou não de equipamentos na área de saúde, educação, transporte, lazer, comunicações, etc. Os dados foram coletados de órgãos de produção estatística, tais como: o Instituto Brasileiro de Geografia e Estatística - IBGE, a base de dados do Sistema Único de Saúde - DATASUS, a Fundação João Pinheiro de Pesquisas do Estado de Minas Gerais - FJP-MG, a base de dados do Instituto de Pesquisas Econômicas Aplicadas - IPEA DATA, o Programa das Nações Unidas para o Desenvolvimento - PNUD, o Instituto de Geociências Aplicadas do Estado de Minas Gerais - IGA, a base de dados do Banco Central, a base de 
dados do Departamento de Estradas de Rodagem de Minas Gerais - DER-MG, entre outros.

\subsection{TRABALHOS DE CAMPO}

Foram realizados trabalhos de campo a fim de enriquecer o banco de dados com informações primárias e também para que houvesse uma confirmação dos dados coletados e, ainda, maior interação dos pesquisadores com o meio a fim de embasá-los para melhores análises a serem feitas acerca dos municípios estudados.

\subsection{COMPONENTES PRINCIPAIS \\ AGRUPAMENTOS}

E

Para a proposição da hierarquia urbana foram utilizadas duas técnicas de estatística multivariada, a análise de componentes principais - ACP e a análise de agrupamentos.

A ACP é uma das técnicas estatísticas multivariadas mais utilizadas que tem por objetivo reduzir a dimensão dos dados originais permitindo a identificação das informações mais importantes em um número menor de fatores, ou componentes principais. Fez-se a opção pela técnica das componentes principais devido à possibilidade de se trabalhar com uma grande variedade e quantidade de dados.

Por meio da ACP criou-se o índice de acessibilidade urbana - IAU e, por meio, da análise de agrupamentos criaram-se os grupos de cidades. Foram utilizados os software NinnaPCA e NinnaCluster. Tais software foram desenvolvidos no Programa de Pós Graduação em Geografia da PUC Minas - Tratamento da Informação Espacial, tema da Tese de Doutorado de Bernardo Jeunon de Alencar, orientado pelos professores Dr. Leônidas Conceição Barroso e Dr. João Francisco de Abreu, sendo disponibilizados pelos seus idealizadores para uso acadêmico.
Existem diversas etapas envolvidas na Análise de Componentes Principais, o leitor desejoso de conhecer a técnica com detalhes pode consultar Alencar (2009).

O software NinnaCluster trabalha com a análise de agrupamentos ou análise de clusters buscando particionar um conjunto de dados formando subconjuntos, ou grupos, de tal forma que os indivíduos, ou observações, presentes em um grupo, tenham alto grau de similaridade entre eles, enquanto que indivíduos pertencentes a diferentes grupos tenham alto grau de dissimilaridade (ALENCAR, 2009).

O referido software oferece quatro opções de métodos de agrupamentos que variam de acordo com o objetivo da pesquisa. Os métodos disponibilizados são:

- método das médias das distâncias ou Average Linkage,

- método da ligação simples, ou Single Linkage, que também é conhecido como método do vizinho mais próximo ou método da distância mínima,

- método da ligação completa, ou Complete Linkage ou método do vizinho mais distante, $\mathrm{e}$

- método K-médias ou K-Means.

No presente trabalho foi escolhido o método K-Means que, segundo Alencar (2009), é um método que procura aumentar a homogeneidade dentro de cada grupo, aumentando também a diferença entre eles. $O$ K-Means é um método de agrupamento nãohierárquico por repartição. Para utilização desse método o pesquisador necessita já ter as hipóteses a respeito do número de conjuntos em seus casos ou variáveis. $\mathrm{Na}$ pesquisa em questão define-se anteriormente o número de classes. O método $k$ Médias produzirá exatamente $\boldsymbol{k}$ diferentes conjuntos com a maior distinção possível entre eles. Para 
conhecer detalhes desta técnica o leitor pode consultar Alencar (2009).

\subsection{REPRESENTAÇÕES CARTOGRÁFICAS}

Foram confeccionados utilizando-se SIG's específicos, mapas coropléticos e isopléticos, que permitiram uma melhor visualização dos resultados, facilitando assim as análises a serem feitas.

Um aspecto importante na confecção das representações cartográficas e na aplicação da técnica K-Means é a estimativa do número de classes. Para elaboração das classes dos cartogramas desse trabalho foi utilizada a fórmula de Sturges, que estima o número recomendável de classes (K) a ser adotado, de acordo com o número total $(n)$ de observações. De acordo com Gerardi (1981) a Fórmula (1) de Sturges se apresenta da seguinte maneira:

$$
K=1+3,3 \log n
$$

Onde:

$\mathrm{K}=\mathrm{n} .^{\circ}$ de classes a serem adotatas.

$\mathrm{n}=\mathrm{n} .^{\circ}$ total de observações.

$\log =$ logaritmo na base 10

Outro tipo de representação cartográfica utilizada nesse trabalho são os cartogramas, que utilizam as figuras geométricas proporcionais. Para que se utilize esse método, deve ser primeiramente definida a forma geométrica a ser trabalhada, para a qual existe uma fórmula especifica, que irá determinar as dimensões dos símbolos proporcionais (DUARTE, 2006). No caso em questão a forma geométrica utilizada será o círculo.

Para se determinar a proporção entre os diversos círculos deve-se, inicialmente, isolar o maior valor dentre os dados (neste caso 1) e determinar, arbitrariamente, o raio de um círculo para representar o dado correspondente (raio $=1 \mathrm{~cm}$ ). O próximo passo é verificar o tamanho do circulo que representará o menor valor da tabela (no caso 0,003). Para alcançar este objetivo deve-se aplicar a Fórmula (2) a seguir:

$$
\mathrm{R}^{\prime}=\sqrt{\mathrm{n}\left(\frac{\mathrm{K}^{2}}{\mathrm{~N}}\right)}
$$

Sendo: $\mathrm{R}^{\prime}=$ raio procurado

$$
\begin{aligned}
& \mathrm{R}^{2}=\text { raio do círculo-base } \\
& \mathrm{n}=\text { Valor estatístico do novo município } \\
& \mathrm{N}=\text { Valor estatístico do círculo-base }
\end{aligned}
$$

\section{RESULTADOS}

Nesta pesquisa a matriz de dados foi obtida através dos dados coletados em diversas fontes de informação tais como o IBGE, a FJP, SEF-MG, PNUD, entre outras. Os dados foram coletados para os vinte e três municípios que compõem a mesorregião do Vale do Mucuri e divididos em cinco grupos temáticos, a saber: acesso a equipamentos de saúde, acesso a equipamentos de educação, economia do município, infraestrutura urbana e uma variável demográfica, perfazendo assim 33 variáveis. O banco de dados completo pode ser consultado no Anexo 1. Do banco de dados original foram selecionadas 15 variáveis para fazerem parte da pesquisa, conforme a Tabela 1. $\mathrm{Na}$ tabela 2 encontram-se as variáveis e os seus valores para cada uma das cidades, segundo o software NinaPCA 
Tabela 2 - Descrição das variáveis utilizadas na pesquisa

\begin{tabular}{|c|c|c|c|}
\hline $\begin{array}{l}\text { Código } \\
\text { da } \\
\text { Variá- } \\
\text { vel }\end{array}$ & Nome da Variável & Fonte de consulta & $\begin{array}{l}\text { Grupo } \\
\text { Temático }\end{array}$ \\
\hline 1 & Nºitos_2005 & $\begin{array}{l}\mathrm{n}^{\circ} \text { total de leitos em hospitais publicos e } \\
\text { privados em diversas especialidades } \\
\text { segundo os cadernos de informação de } \\
\text { saude do DATASUS } 2007\end{array}$ & \multirow[b]{2}{*}{$\begin{array}{l}\text { Equipamentos } \\
\text { de Saúde }\end{array}$} \\
\hline 2 & $\begin{array}{l}\text { No Estabelecimentos de } \\
\text { Saúde }\end{array}$ & $\begin{array}{l}\mathrm{n}^{\circ} \text { de estabelecimentos de saude } \\
\text { (hospitais, postos de saude, clinicas, } \\
\text { policlinicas, etc) publicos e privados } \\
\text { conforme cadernos de informação de } \\
\text { saude do DATASUS } 2007\end{array}$ & \\
\hline 3 & $\begin{array}{l}N^{\circ} \text { Matrículas Pré- } \\
\text { primário }\end{array}$ & $\begin{array}{l}\text { Informações segundo a } \\
\text { SEE/SI/SIE/Diretoria de Informações } \\
\text { Educacionais Maio/08 }\end{array}$ & \multirow{6}{*}{$\begin{array}{l}\text { Equipamentos } \\
\text { de Educação }\end{array}$} \\
\hline 4 & $\begin{array}{l}\text { № Matriculas Ensino } \\
\text { Fundamental }\end{array}$ & $\begin{array}{l}\text { Informações segundo a } \\
\text { SEE/SI/SIE/Diretoria de Informações } \\
\text { Educacionais Maio/08 }\end{array}$ & \\
\hline 5 & $\begin{array}{l}N^{\circ} \text { Matrículas Ensino } \\
\text { Superior. }\end{array}$ & $\begin{array}{l}\text { Informações segundo a } \\
\text { SEE/SI/SIE/Diretoria de Informações } \\
\text { Educacionais Maio/08 }\end{array}$ & \\
\hline 6 & $\begin{array}{l}N^{\circ} \text { Estabelecimentos } \\
\text { Educacionais com Pré- } \\
\text { primário }\end{array}$ & $\begin{array}{l}\text { Informações segundo a } \\
\text { SEE/SI/SIE/Diretoria de Informações } \\
\text { Educacionais Maio/08 }\end{array}$ & \\
\hline 7 & $\begin{array}{l}\mathrm{N}^{\circ} \text { Estabelecimentos } \\
\text { Educacionais com } \\
\text { Ensino Fundamental }\end{array}$ & $\begin{array}{l}\text { Informações segundo a } \\
\text { SEE/SI/SIE/Diretoria de Informações } \\
\text { Educacionais Maio/08 }\end{array}$ & \\
\hline 8 & $\begin{array}{l}N^{\circ} \text { Estabelecimentos } \\
\text { com Ensino Superior }\end{array}$ & $\begin{array}{l}\text { Informações segundo a } \\
\text { SEE/SI/SIE/Diretoria de Informações } \\
\text { Educacionais Maio/08 }\end{array}$ & \\
\hline 9 & PIB total & $\begin{array}{l}\text { PIB valores correntes segundo o IBGE } \\
2006 .\end{array}$ & \multirow{3}{*}{$\begin{array}{l}\text { Economia } \\
\text { Municipal }\end{array}$} \\
\hline 10 & Arrecadação Municipal & SEF - Arrecadação municipal - nov. 2009 & \\
\hline 11 & $\begin{array}{l}N^{0} \text { empregos no } \\
\text { comércio e Serviços }\end{array}$ & $\begin{array}{l}\mathrm{n}^{\circ} \text { de pessoas empregadas por setor - } \\
\text { Ministerio doTrabalho e Emprego - } 2007\end{array}$ & \\
\hline 12 & $\begin{array}{l}\text { Frota de Ônibus e } \\
\text { Micro Ônibus }\end{array}$ & $\begin{array}{l}\text { Frota de veiculos composta por Micro- } \\
\text { ônibus, e Ônibus segundo Ministério das } \\
\text { Cidades, Departamento Nacional de } \\
\text { Trânsito - DENATRAN, Sistema Nacional } \\
\text { de Registro de Veículos/RENAVAM, } \\
\text { Sistema Nacional de Estatística de } \\
\text { Trânsito/SINET, jan/2008 }\end{array}$ & \multirow[t]{3}{*}{$\begin{array}{l}\text { Serviços } \\
\text { Urbanos }\end{array}$} \\
\hline 13 & No Agências Bancarias & $\begin{array}{l}\mathrm{n}^{\circ} \text { de agencias por municipio segundo o } \\
\text { Banco Central. julho/2009 }\end{array}$ & \\
\hline 14 & No Hotéis & $\begin{array}{l}\text { Hoteis e pousadas por municipios } \\
\text { segundo ABIH - Associação Brasileira da } \\
\text { Indústria de Hoteis - } 2000\end{array}$ & \\
\hline 15 & População Urbana & IBGE 2007 & Demografia \\
\hline
\end{tabular}

Fonte: APOLINÁRIO, 2010. 

Tabela 2 Dados coletados e suas respectivas variáveis. Fonte: APOLINÁRIO, 2010.

\begin{tabular}{|c|c|c|c|c|c|c|c|c|c|c|c|c|c|c|c|}
\hline Caso & Var.1 & Var.2 & Var.3 & Var.4 & Var.5 & Var.6 & Var.7 & Var.8 & Var.9 & Var.10 & Var.11 & Var.12 & Var.13 & Var.14 & Var.15 \\
\hline 3100906 & \begin{tabular}{|l}
87 \\
\end{tabular} & 8 & \begin{tabular}{|r|}
351 \\
\end{tabular} & \begin{tabular}{|l|}
3751 \\
\end{tabular} & \begin{tabular}{|l|}
119 \\
\end{tabular} & 5 & 31 & 0 & 73945 & 1768644 & 474 & 38 & 2 & 3 & 14073 \\
\hline 3104700 & 42 & 6 & 184 & 2794 & 0 & 7 & 17 & 0 & 68437 & 709112 & 90 & 32 & 1 & 2 & 7121 \\
\hline 3106606 & 0 & 4 & 71 & 994 & 0 & 1 & 5 & 0 & 19874 & 399180 & 9 & 5 & 0 & 0 & 2521 \\
\hline 3113701 & 59 & 21 & 423 & 3686 & 49 & 5 & 17 & 0 & 144729 & 3741763 & 697 & 64 & 3 & 2 & 13289 \\
\hline 3115458 & 0 & 4 & 149 & 2039 & 0 & 2 & 19 & 0 & 24757 & 248550 & 74 & 21 & 0 & 0 & 1546 \\
\hline 3120151 & 0 & 4 & 126 & 1417 & 0 & 1 & 9 & 0 & 27363 & 107211 & 12 & 13 & 0 & 0 & 3512 \\
\hline 3126752 & 0 & 1 & 65 & 1218 & 0 & 1 & 10 & 0 & 22929 & 108404 & 3 & 11 & 0 & 0 & 2213 \\
\hline 3126802 & 0 & 3 & 162 & 1381 & 0 & 2 & 7 & 0 & 27166 & 232945 & 11 & 11 & 0 & 0 & 1867 \\
\hline 3127057 & 0 & 6 & 112 & 968 & 0 & 1 & 7 & 0 & 16178 & 87390 & 10 & 6 & 0 & 0 & 2953 \\
\hline 3132305 & 26 & 3 & 147 & 3009 & 0 & 2 & 22 & 0 & 41391 & 438579 & 71 & 18 & 1 & 1 & 4846 \\
\hline 3137007 & 23 & 6 & 143 & 3848 & 76 & 1 & 32 & 0 & 45482 & 427999 & 62 & 31 & 1 & 0 & 4206 \\
\hline 3138906 & 58 & 4 & 173 & 1379 & 40 & 2 & 5 & 0 & 30083 & 518193 & 79 & 7 & 1 & 0 & 5714 \\
\hline 3139201 & 75 & 11 & 399 & 4431 & 0 & 6 & 21 & 0 & 70207 & 1257489 & 268 & 36 & 1 & 2 & 10912 \\
\hline 3144300 & 108 & 22 & 978 & 7182 & 1251 & 18 & 23 & 1 & 300053 & 9834921,48 & 2427 & 205 & 6 & 8 & 37253 \\
\hline 3145356 & 0 & 4 & 197 & 2284 & 0 & 3 & 16 & 0 & 30209 & 1324875,59 & 233 & 18 & 0 & 0 & 4175 \\
\hline 3146206 & 0 & 3 & 137 & 1767 & 0 & 1 & 20 & 0 & 23313 & 131727 & 30 & 7 & 0 & 0 & 3965 \\
\hline 3148509 & 44 & 6 & 196 & 1542 & 0 & 3 & 6 & 0 & 37393 & 607879 & 137 & 17 & 1 & 1 & 5390 \\
\hline 3152402 & 34 & 8 & 287 & 3298 & 54 & 6 & 20 & 0 & 55944 & 900507 & 183 & 23 & 1 & 1 & 8752 \\
\hline 3157658 & 0 & 4 & 120 & 1423 & 0 & 1 & 8 & 0 & 19518 & 141381 & 4 & 7 & 0 & 0 & 3305 \\
\hline 3166709 & 0 & 6 & 275 & 1674 & 0 & 4 & 10 & 0 & 59528 & 1163005,61 & 110 & 24 & 1 & 1 & 6847 \\
\hline 3165552 & 0 & 3 & 117 & 2710 & 48 & 3 & 14 & 0 & 26660 & 102585 & 22 & 16 & 0 & 0 & 2465 \\
\hline 3168606 & 417 & 65 & 2371 & 25134 & 5796 & 46 & 98 & 4 & 883761 & 43866609,65 & 12102 & 260 & 11 & 27 & 102474 \\
\hline 3170305 & 0 & 4 & 83 & 574 & 0 & 2 & 3 & 0 & 14475 & 71282,04 & 9 & 3 & 0 & 0 & 1591 \\
\hline & & & & & & & & & & & & & & & \\
\hline & Var.1 & \multicolumn{3}{|c|}{$N^{\circ}$ Leitos } & & Var.6 & \multicolumn{4}{|c|}{$\begin{array}{l}\mathrm{N}^{\circ} \text { Estabelecimentos Educacionais com } \\
\text { Pré-primário }\end{array}$} & & Var.11 & \multicolumn{3}{|c|}{$\begin{array}{c}N^{\circ} \text { empregos no comércio } \\
\text { e Serviços }\end{array}$} \\
\hline & Var.2 & \multicolumn{3}{|c|}{$\begin{array}{l}\text { No Estabelecimentos de } \\
\text { Saúde }\end{array}$} & & Var.7 & \multicolumn{4}{|c|}{$\begin{array}{c}N^{\circ} \text { Estabelecimentos Educacionais com } \\
\text { Ensino Fundamental }\end{array}$} & & Var.12 & \multicolumn{3}{|c|}{$\begin{array}{c}\text { Frota de Onibus e Micro } \\
\text { Onibus }\end{array}$} \\
\hline & Var.3 & \multicolumn{3}{|c|}{ № Matrículas Pré-primário } & & Var.8 & \multicolumn{4}{|c|}{$\mathrm{N}^{\circ}$ Estabelecimentos com Ensino Superior } & & Var.13 & \multicolumn{3}{|c|}{ No Agências Bancarias } \\
\hline & Var.4 & \multicolumn{3}{|c|}{$\begin{array}{l}\text { No Matriculas Ensino } \\
\text { Fundamental }\end{array}$} & & Var.9 & \multicolumn{4}{|c|}{ PIB total } & & Var.14 & \multicolumn{3}{|c|}{$N^{\circ}$ Hoteis } \\
\hline & Var.5 & \multicolumn{3}{|c|}{$\begin{array}{l}\text { No Matrículas Ensino } \\
\text { Superior. }\end{array}$} & & Var.10 & \multicolumn{4}{|c|}{ Arrecadação Municipal } & & Var.15 & \multicolumn{3}{|c|}{ População Urbana } \\
\hline
\end{tabular}


No presente trabalho foram utilizados os escores relativos à primeira componente principal, já que a mesma explicou $96,13 \%$ da variabilidade dos dados. Estes escores representam o Índice de Acessibilidade Urbana (Tabela 3).

Tabela 3: Dados padronizados do Índice de Acessibilidade Urbana.

\begin{tabular}{|l|l|c|}
\hline Codigo & \multicolumn{1}{|c|}{ Nome } & \multicolumn{1}{c|}{ Escore } \\
\hline $\mathbf{3 1 0 0 9 0 6}$ & Águas Formosas & 0,06956 \\
\hline $\mathbf{3 1 0 4 7 0 0}$ & Ataléia & $-0,14401$ \\
\hline $\mathbf{3 1 0 6 6 0 6}$ & Bertópolis & $-0,43307$ \\
\hline $\mathbf{3 1 1 3 7 0 1}$ & Carlos Chagas & 0,16113 \\
\hline $\mathbf{3 1 1 5 4 5 8}$ & Catuji & $-0,33589$ \\
\hline $\mathbf{3 1 2 0 1 5 1}$ & Crisólita & $-0,39313$ \\
\hline $\mathbf{3 1 2 6 7 5 2}$ & Franciscópolis & $-0,42541$ \\
\hline $\mathbf{3 1 2 6 8 0 2}$ & Frei Gaspar & $-0,39985$ \\
\hline $\mathbf{3 1 2 7 0 5 7}$ & Fronteira dos Vales & $-0,41151$ \\
\hline $\mathbf{3 1 3 2 3 0 5}$ & Itaipé & $-0,24164$ \\
\hline $\mathbf{3 1 3 7 0 0 7}$ & Ladainha & $-0,18553$ \\
\hline $\mathbf{3 1 3 8 9 0 6}$ & Machacalis & $-0,31082$ \\
\hline $\mathbf{3 1 3 9 2 0 1}$ & Malacacheta & $-0,00462$ \\
\hline $\mathbf{3 1 4 4 3 0 0}$ & Nanuque & 1,13810 \\
\hline $\mathbf{3 1 4 5 3 5 6}$ & Novo Oriente de Minas & $-0,30800$ \\
\hline $\mathbf{3 1 4 6 2 0 6}$ & Ouro Verde de Minas & $-0,35977$ \\
\hline $\mathbf{3 1 4 8 5 0 9}$ & Pavão & $-0,27048$ \\
\hline $\mathbf{3 1 5 2 4 0 2}$ & Poté & $-0,12881$ \\
\hline $\mathbf{3 1 5 7 6 5 8}$ & Santa Helena de Minas & $-0,40722$ \\
\hline $\mathbf{3 1 6 6 7 0 9}$ & Serra dos Aimorés & $-0,24666$ \\
\hline $\mathbf{3 1 6 5 5 5 2}$ & Setubinha & $-0,34725$ \\
\hline $\mathbf{3 1 6 8 6 0 6}$ & Teófilo Otoni & 4,43147 \\
\hline $\mathbf{3 1 7 0 3 0 5}$ & Umburatiba & $-0,44655$ \\
\hline & \\
\hline
\end{tabular}

Fonte: APOLINÁRIO, 2010.

Foram definidos cinco agrupamentos e o software NinnaCluster agrupou os municípios da seguinte maneira:
Tabela 4: Grupos de municípios gerados pelo software NinaCluster.

\begin{tabular}{|c|c|}
\hline Cluster & Município \\
\hline \multirow{10}{*}{ Cluster \# 1} & Umburatiba \\
\hline & Bertópolis \\
\hline & Franciscópolis \\
\hline & Fronteira dos Vales \\
\hline & Santa Helena de Minas \\
\hline & Frei Gaspar \\
\hline & Crisólita \\
\hline & Ouro Verde de Minas \\
\hline & Setubinha \\
\hline & Catuji \\
\hline Cluster \# 2 & Teófilo Otoni \\
\hline \multirow{8}{*}{ Cluster \# 3} & Machacalis \\
\hline & Pavão \\
\hline & Novo Oriente de Minas \\
\hline & Serra dos Aimorés \\
\hline & Itaipé \\
\hline & Ladainha \\
\hline & Ataléia \\
\hline & Poté \\
\hline \multirow{3}{*}{ Cluster \# 4} & Malacacheta \\
\hline & Águas Formosas \\
\hline & Carlos Chagas \\
\hline Cluster \# 5 & Nanuque \\
\hline
\end{tabular}

Fonte: APOLINÁRIO, 2010.

Após definidos os grupos de municípios chega-se à fase final do trabalho, o mapeamento dos resultados com base nos cálculos efetuados. O software utilizado para confecção das representações cartográficas foi Maplnfo 9.0. Após gerar tabelas em formato Excel, as mesmas foram exportadas para o MapInfo, onde geraram mapas retratando uma proposta de hierarquia urbana na mesorregião do Vale do Mucuri.

Foram elaborados dois cartogramas para representar os resultados obtidos. 
O primeiro (Mapa 2) faz uso do método coroplético, que consiste em apresentar os resultados em uma ordem crescente dos valores relativos agrupados em classes, que serão transcritas por uma ordem visual. Aplicando a fórmula de Sturges para os municípios que compõem a Mesorregião do Vale do Mucuri, e que são em número de 23 , foi obtido $K=5,49$, ficando então convencionado que os cartogramas tivessem cinco classes.

No segundo cartograma foi utilizado o método das figuras geométricas proporcionais, que tem como objetivo demonstrar quantidades absolutas em áreas distintas quando se tem interesse em visualizar a proporção entre o fenômeno. Este método mobiliza a única variação visual que transcreve fielmente a noção de quantidade em proporção: 0 tamanho (MARTINELLI, 2006). Os símbolos mais utilizados para traduzir graficamente valores estatísticos são o triângulo, o quadrado, e o círculo, sendo este último o mais utilizado (DUARTE, 2006).

Para que não fossem utilizados nessa representação valores negativos, foi realizada uma padronização dos dados fazendo com que todos tivessem valores que variassem de 0 a 1.

Para essa operação foi utilizada a seguinte Fórmula (3):

$$
\frac{\text { Vr. Ubs. - Mencr Vr. }}{\text { Maiur V. - Menor VI. }}
$$

Utilizando-se a Fórmula (3) com os dados do índice de acessibilidade urbana temos os dados mostrados na tabela 5 .

Posteriormente foram efetuados os cálculos para os demais municípios e desenhados os respectivos círculos proporcionais.
Tabela 5 - Dados padronizados do Índice de Acessibilidade Urbana.

\begin{tabular}{|r|l|l|}
\hline Código & \multicolumn{1}{|c|}{ Nome } & $\begin{array}{l}\text { Escore } \\
\text { Padronizado }\end{array}$ \\
\hline $\mathbf{3 1 0 0 9 0 6}$ & Águas Formosas & 0,106 \\
\hline $\mathbf{3 1 0 4 7 0 0}$ & Ataléia & 0,062 \\
\hline $\mathbf{3 1 0 6 6 0 6}$ & Bertópolis & 0,003 \\
\hline $\mathbf{3 1 1 3 7 0 1}$ & Carlos Chagas & 0,125 \\
\hline $\mathbf{3 1 1 5 4 5 8}$ & Catuji & 0,023 \\
\hline $\mathbf{3 1 2 0 1 5 1}$ & Crisólita & 0,011 \\
\hline $\mathbf{3 1 2 6 7 5 2}$ & Franciscópolis & 0,004 \\
\hline $\mathbf{3 1 2 6 8 0 2}$ & Frei Gaspar & 0,010 \\
\hline $\mathbf{3 1 2 7 0 5 7}$ & Fronteira dos Vales & 0,007 \\
\hline $\mathbf{3 1 3 2 3 0 5}$ & Itaipé & 0,042 \\
\hline $\mathbf{3 1 3 7 0 0 7}$ & Ladainha & 0,054 \\
\hline $\mathbf{3 1 3 8 9 0 6}$ & Machacalis & 0,028 \\
\hline $\mathbf{3 1 3 9 2 0 1}$ & Malacacheta & 0,091 \\
\hline $\mathbf{3 1 4 4 3 0 0}$ & Nanuque & 0,325 \\
\hline $\mathbf{3 1 4 5 3 5 6}$ & $\begin{array}{l}\text { Novo Oriente de } \\
\text { Minas }\end{array}$ & 0,028 \\
\hline & Ouro Verde de & \\
\hline $\mathbf{3 1 4 6 2 0 6}$ & Minas & 0,018 \\
\hline $\mathbf{3 1 4 8 5 0 9}$ & Pavão & 0,036 \\
\hline $\mathbf{3 1 5 2 4 0 2}$ & Poté & 0,065 \\
\hline & Santa Helena de & 0,008 \\
\hline $\mathbf{3 1 5 7 6 5 8}$ & Minas & 0,041 \\
\hline $\mathbf{3 1 6 6 7 0 9}$ & Serra dos Aimorés & 0,020 \\
\hline $\mathbf{3 1 6 5 5 5 2}$ & Setubinha & 1,000 \\
\hline $\mathbf{3 1 6 8 6 0 6}$ & Teófilo Otoni & 0,000 \\
\hline $\mathbf{3 1 7 0 3 0 5}$ & Umburatiba & \\
& & 0,0 \\
\hline
\end{tabular}

Fonte: APOLINÁRIO, 2010.

O Mapa 3 representa o índice de acessibilidade urbana através dos círculos proporcionais.

Utilizando o método das figuras geométricas proporcionais, tem-se:

$\mathrm{R}^{\prime}=$ raio procurado

$\mathrm{R}^{2}=$ raio do círculo-base $(1 \mathrm{~cm}-$ município de Teófilo Otoni)

$\mathrm{n}=$ Valor estatístico do novo município $(0,003-$ município de Bertópolis)

$\mathrm{N}=$ Valor estatístico do círculo-base (1,00 - Teófilo

Otoni)

Aplicando a fórmula tem-se:

$$
\mathrm{R}^{\prime}=\sqrt{0.003\left(\frac{1^{2}}{1}\right)}=0,05 \mathrm{~cm} .
$$




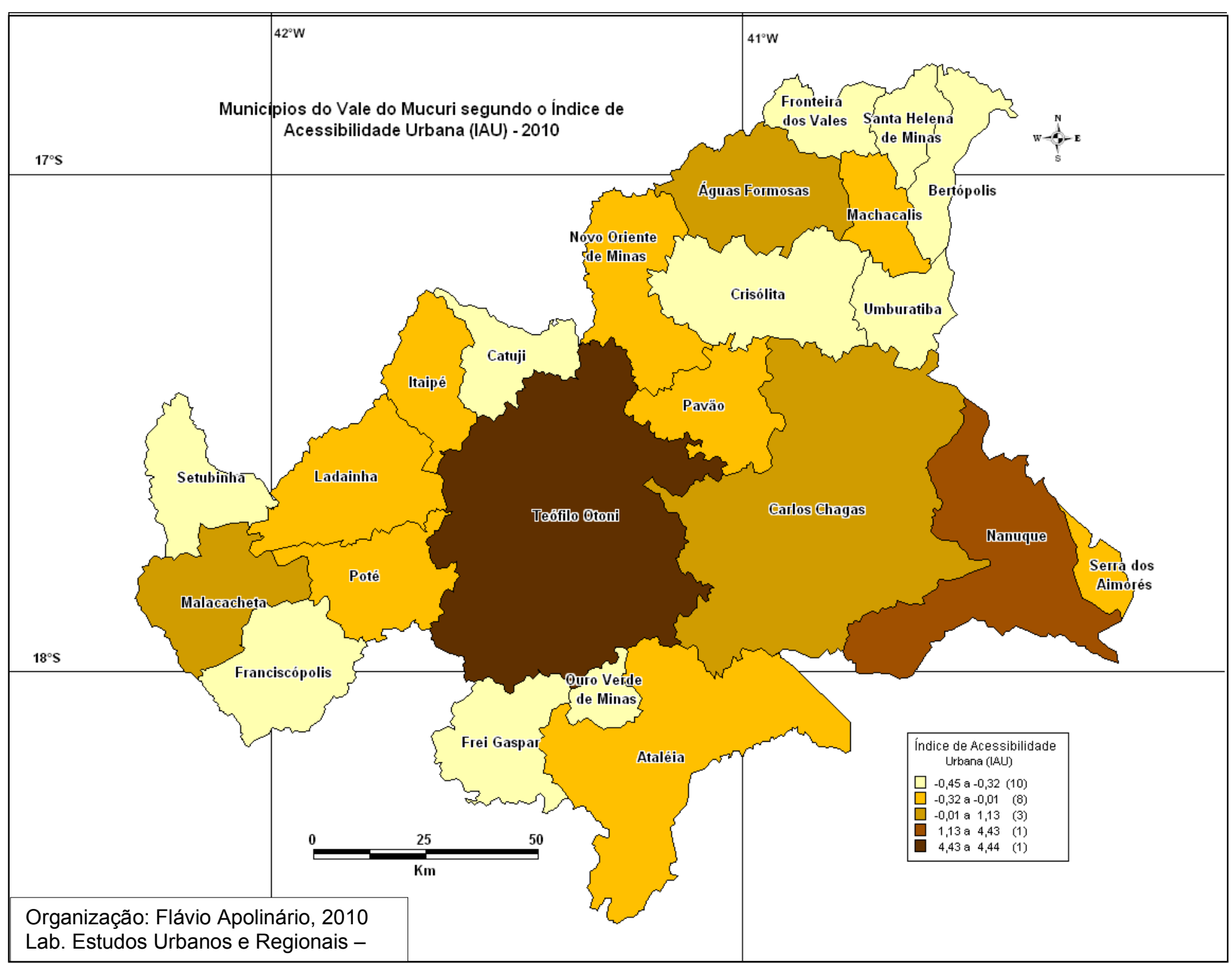

Mapa 2: Municípios da Mesorregião do Vale do Mucuri segundo o Índice de Acessibilidade Urbana (IAU). Fonte: APOLINÁRIO, 2010.

e-xacta, Belo Horizonte, v. 5, n 2, p. 19-32. (2012). Editora UniBH. Disponível em: www.unibh.br/revistas/exacta/ 


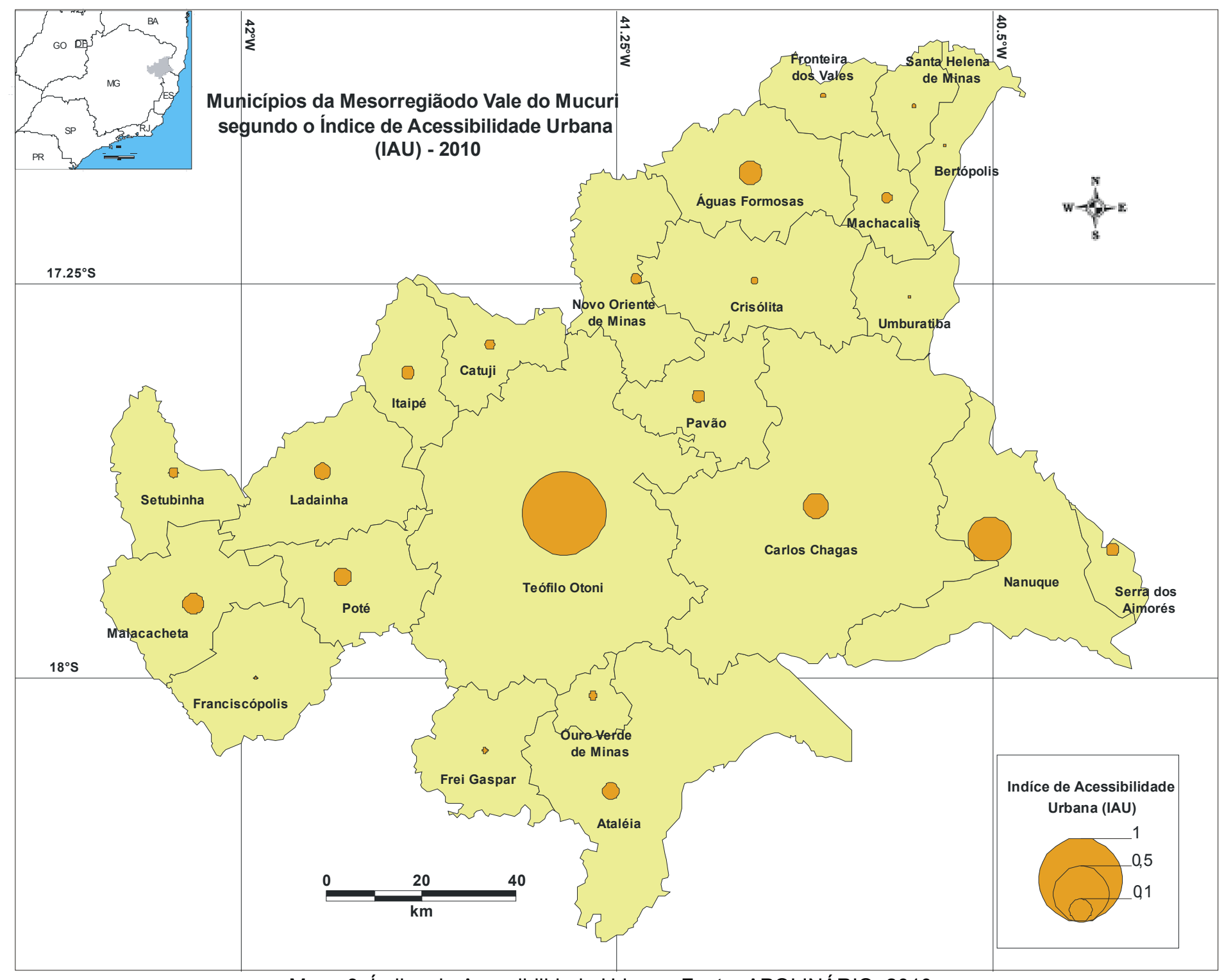

Mapa 3: Índice de Acessibilidade Urbana. Fonte: APOLINÁRIO, 2010.

e-xacta, Belo Horizonte, v. 5, n 2, p. 19-32. (2012). Editora UniBH. 
Analisando a representação cartográfica apresentada, pode-se identificar alguns níveis hierárquicos, a saber:

$1^{0}$ Nível - Isolada no topo da hierarquia da mesorregião do Vale do Mucuri encontra-se a cidade de Teófilo Otoni. Esta cidade tem a população urbana mais expressiva da região e dispõe de uma completa infraestrutura de serviços que polariza todas as cidades da região. Teófilo Otoni se diferencia das demais cidades do Vale do Mucuri tanto no aspecto quantitativo, quanto no qualitativo de seus serviços. A cidade é servida por grandes redes de comércio, tanto varejista, quanto atacadista, tem uma rede bancária diversificada, composta por bancos públicos e privados, seus equipamentos na área da saúde são os mais completos de toda a região, fazendo com que pessoas de diversas cidades busquem atendimentos, principalmente os especializados, em Teófilo Otoni. $\mathrm{Na}$ área de educação a cidade se destaca tanto pela quantidade de equipamentos, quanto pela infraestrutura que eles oferecem, sendo ela bem superior à das demais cidades da região. Os níveis de ensino também são um diferencial em relação aos demais municípios.

20 Nível - Nanuque ocupa, também isolada, a segunda posição do ranking de cidades do Vale do Mucuri. Esta cidade não possui uma gama de serviços tão completa quanto à e Teófilo Otoni, mas também não pode ser agrupada com as cidades que estão em um nível hierárquico inferior a ela, deixando-a em um nível intermediário.

$3^{0}$ Nível - Águas Formosas, Carlos Chagas e Malacacheta encontram-se em um mesmo nível, que pode ser caracterizado como de "Centros Emergentes". Essas três cidades devem sua posição diferenciada das demais, sobretudo por sua posição geográfica. Carlos Chagas faz a intermediação entre Teófilo Otoni e Nanuque, enquanto Águas Formosas polariza os serviços no extremo Norte da mesorregião. Da mesma forma Malacacheta também polariza o extremo Oeste da mesorregião. Estas duas últimas cidades estão fora da abrangência dos dois principais eixos viários da mesorregião, as BR's 342 e 418 e por isso oferecem seus serviços às cidades do seu entorno.

$4^{0}$ Nível - Ataléia, Itaipé, Ladainha, Machacalis, Novo Oriente de Minas, Pavão, Poté e Serra dos Aimorés fazem parte do grupo de cidades que se encontram no nível inferior da hierarquia, no entanto têm uma infraestrutura de serviços ligeiramente mais desenvolvida que as demais.

$5^{\circ}$ Nivel - Bertópolis, Catuji, Crisólita, Franciscópolis, Frei Gaspar, Fronteira dos Vales, Ouro Verde de Minas, Santa Helena de Minas, Setubinha e Umburatiba são as cidades com a infraestrutura de serviços mais elementar da região.

\section{AnÁlise dos Resultados}

Observando-se a tipologia das cidades, sua posição geográfica e as interações que ocorrem entre elas, comprovou-se que a Rede Urbana da mesorregião do Vale do Mucuri, encontra-se, ainda, bastante desarticulada no que diz respeito à polarização de serviços e às áreas de influência que esses serviços poderiam gerar. Uma rede urbana demanda que as cidades que a compõem ocupem níveis hierárquicos distintos, garantindo assim uma articulação funcional dinâmica entre elas. Isto não acontece na região em estudo, pois os níveis hierárquicos são bastante reduzidos tendo, em um nível isolado, a cidade de Teófilo Otoni, em outro, a cidade de Nanuque, seguida de um terceiro nível formado por três cidades: Carlos Chagas, Malacacheta e Águas formosas. As outras dezoito cidades se dividem em dois níveis onde as diferenças entre eles estão muito mais no nível quantitativo do que qualitativo. $\mathrm{O}$ baixo número de cidades nos níveis iniciais da rede 
demonstra, mais uma vez, a falta de articulação entre as cidades e a incipiência dessa rede urbana.

Para que uma rede urbana esteja bem estruturada é necessário também que exista uma diferenciação interurbana entre as cidades, criando uma articulação funcional. Os níveis hierárquicos da rede devem ser bem definidos e devem estar articulados entre si, para que haja um funcionamento dinâmico.

No caso do Vale do Mucuri os níveis hierárquicos estão pouco articulados, já que existem poucas cidades nos níveis mais elevados da hierarquia e que a diferenciação entre essas e os níveis posteriores são muito grandes, não vislumbrando uma ascensão, pelo menos a curto prazo, de municípios dos níveis intermediários. Outro aspecto negativo com relação à rede de cidades do Vale do Mucuri é a grande quantidade de municípios que se aglomeram no último nível da hierarquia, onde se encontram as cidades mais subequipadas da mesorregião.

Fazendo uma análise espacial da mesorregião podese identificar claramente um eixo de integração que se estende no sentido oeste-leste, do qual fazem parte as cidades que estão nos níveis mais altos da hierarquia: Teófilo Otoni e Nanuque, tendo como ligação entre elas a cidade de Carlos Chagas caracterizada como um Centro Emergente. Outra cidade classificada como Centro Emergente e localizada no extremo oeste da mesorregião é Malacacheta, que completa o eixo oeste-leste.

No extremo Norte da mesorregião, encontra-se a cidade de Águas Formosas, que também foi classificada, nesse trabalho, como um "Centro Emergente". Essa classificação pode ser explicada pela própria localização geográfica, que se encontra marginalizada em relação aos principais eixos viários da região, a BR- 418, conhecida como "Rodovia do Boi", que corta a região no sentido oeste-leste e a BR-342 conhecida como "Rio-Bahia", fazendo com que essa cidade exerça sua centralidade na porção setentrional da região.

Feitas essas análises pode-se afirmar que a rede de cidades do Vale do Mucuri ainda encontra-se em estado bastante incipiente, devido principalmente à falta de níveis hierárquicos e à baixa diferenciação entre as tipologias das cidades da rede, o que faz com que o aspecto da complementaridade alcance níveis bastante baixos entre os centros estudados.

Tanto na pesquisa efetuada por Amorim Filho (1990), quanto no estudo atual, sobre a rede de cidades do Vale do Mucuri, pode-se constatar a posição de "Primazia Urbana" ocupada pela cidade de Teófilo Otoni.

O conceito de primazia urbana foi introduzido pelo geógrafo americano Mark Jefferson nos anos 30 . Segundo Jefferson (1939) a cidade primaz é desproporcionalmente grande, e bastante carregada de sentimento regional. Essa cidade é pelo menos duas vezes tão grande quanto a segunda maior cidade da rede, e também duas vezes mais significativa.

Ainda segundo Jefferson (1939) a primazia de uma cidade pode estar ligada a alguns condicionantes, que ao longo dos estudos se mostraram presentes no caso de Teófilo Otoni:

- História de ocupação e colonização - Como já foi visto no início deste trabalho, 21 dos 23 municípios da mesorregião são oriundos do grande território que a cidade de Teófilo Otoni ocupava.

- Concentração de Investimentos - A história nos mostra que devido a seu fundador, Teófilo Benedito Otoni, esse município sempre recebeu maiores investimentos que as outras cidades da região.

- Estruturas econômico-espaciais muito simples - a análise dos dados relativos aos 
municípios confirma a simplicidade das estruturas econômico-espaciais da região estudada.

- $\quad$ Presença de pequenas populações - Dos 23 municípios estudados, 13 têm menos de 5.000 habitantes, 5 têm menos de 10.000 habitantes e 3 têm menos de 15.000 habitantes.

- Baixos níveis de renda - o Vale do Mucuri tem a terceira pior renda per capita do estado se comparado com as outras onze mesorregiões mineiras.

- Dependência econômica da agricultura - A maioria dos municípios estudados tem como sua principal atividade econômica o extrativismo mineral e vegetal, a pecuária extensiva e a as atividades agrícolas, bastante desorganizadas.

Diante de todas essas constatações pode-se afirmar a posição de primazia urbana que Teófilo Otoni ocupa em relação à rede de cidades do Vale do Mucuri.

É importante destacar que o estudo em questão fez uso de várias fontes secundárias de dados, levantadas junto aos órgãos de produção estatísticas de diversas esferas públicas e privadas, servindo para a formação do banco de dados, que subsidiou grande parte das etapas da pesquisa. Após esse levantamento foram executados trabalhos de campo que foram de suma importância para comprovar, de forma empírica, os dados coletados em gabinete. Além dessas confirmações os trabalhos de campo foram primordiais para que fossem visualizados os aspectos qualitativos dos serviços oferecidos em cada um dos municípios visitados, bem como permitir ao pesquisador uma maior interação com a realidade vivida pelos habitantes da região estudada.

O aporte teórico da Geografia Urbana aliado às tecnologias dos SIG's e das técnicas de estatísticas multivariadas, utilizadas nesse trabalho, permitiram uma confirmação sobre as observações empíricas realizadas através dos trabalhos de campo na área de estudo.

A pretensão dos autores é que em estudos futuros os dados adquiridos, bem como a experiência acerca da área de estudo, possam ser utilizados em novas pesquisas, que visem contribuir para um melhor conhecimento da região do Vale do Mucuri.

\section{REFERÊNCIAS}

ALENCAR, Bernardo Jeunon de. A análise multivariada no tratamento da informação espacial: uma abordagem matemáticocomputacional em análise de agrupamentos e análise de componentes principais. 2009. 200 f. Tese (Doutorado em Geografia - Tratamento da Informação Espacial)-Pontifícia Universidade Católica de Minas Gerais - PUC Minas. Belo Horizonte, 2000.

AMORIM FILHO, O.B. A rede urbana da Bacia do Mucuri. Revista Geografia e Ensino. Belo Horizonte, 3(1): 26-36, 1990.
APOLINÁRIO, Flávio. A rede urbana da Mesorregião do Vale do Mucuri: uma proposta de hierarquização por meio de técnicas de estatística multivariada. Dissertação (Mestrado em Geografia Tratamento da Informação Espacial)-Pontifícia Universidade Católica de Minas Gerais, PUC Minas. Belo Horizonte, 2010. Disponível em: <http://www.biblioteca.pucminas.br/teses/TratInfEspa cial_ApolinarioF_1.pdf>.

DUARTE, P. A. Fundamentos de Cartografia. Florianópolis: Ed. da UFSC, 2006. 
GERARDI, L. H. O. Quantificação em Geografia. 1. ed. São Paulo: Editora Difel, 1981.161 p.

IBGE - Instituto Brasileiro de Geografia e Estatística. Regiões de Influência das Cidades. 2007.

IBGE - Instituto Brasileiro de Geografia e Estatística. Contagem da População 2007; Área territorial oficial. Rio de Janeiro. Disponível em: <http://www.ibge.gov.br/home/geociencias/cartografi a/default_territ_area.shtm>. Acesso em: mar. 2008.

IBGE - Instituto Brasileiro de Geografia e Estatística. Resolução $N^{\circ}$ 05, de 10 de outubro de 2002, Publicado no Diário Oficial da União $\mathbf{N}^{\circ} 198$ Seção 1, de 11/10/2002, p. 48-65.
IBGE - Instituto Brasileiro de Geografia e Estatística. Divisão Regional do Brasil em Mesorregiões e Microrregiões Geográficas. v. 1, Rio de Janeiro, 1990.

JEFFERSON, Mark. The Law of the Primate City, in Geographical Review. v.29, p. 226-232, April 1939.

MARTINELLI, Marcelo. O ensino da cartografia temática. In: CASTELLAR, Sonia. (Org.) Educação Geográfica: Teorias e Práticas Docentes. São Paulo: Contexto, 2006. p. 51-65. 